\title{
3D Reproduction of a Snow Crystal by Stereolithography*
}

\author{
Jun'ichi TAMAKI**, Satoshi YANAGI**, Yuya AOKI**, Akihiko KUBO**, \\ Takao KAMEDA** and A. M. M. Sharif ULLAH** \\ ${ }^{* * K i t a m i ~ I n s t i t u t e ~ o f ~ T e c h n o l o g y, ~}$ \\ 165 Koen-cho, Kitami, Hokkaido, 090-8507, Japan \\ E-mail: tamaju@mail.kitami-it.ac.jp
}

\begin{abstract}
A new method was proposed for replicating snow crystals that uses light-curing resin containing no harmful substances, as the replicating material, and the $3 \mathrm{D}$ reproduction of a snow crystal by stereolithography was conducted. It was found that a UV light irradiation density of at least $0.6 \mathrm{~mW} / \mathrm{cm}^{2}$ was required to complete the light-hardening reaction within $15 \mathrm{~min}$ when polyene/polythiol resin (NOA81) was used as the light-curing resin. When the atmospheric temperature was $0{ }^{\circ} \mathrm{C}$, the maximum temperature rise due to the light-hardening reaction was $4.2{ }^{\circ} \mathrm{C}$ at an irradiation density of $1.0 \mathrm{~mW} / \mathrm{cm}^{2}$. This suggests that the initial temperature of the light-curing resin must be approximately $-5{ }^{\circ} \mathrm{C}$ to prevent the snow crystal from melting when an irradiation density of $1.0 \mathrm{~mW} / \mathrm{cm}^{2}$ is applied at an atmospheric temperature of below $0{ }^{\circ} \mathrm{C}$. This replication method has sufficient accuracy to reconstruct the 3D shape of a snow crystal. The 3D reproduction of a snow crystal by stereolithography was conducted by transforming the CSV-formatted 3D profile height data to STL-formatted CAD data.
\end{abstract}

Key words: Snow Crystal, 3D Reproduction, Light-Curing Resin, Irradiation Density, Light-Hardening Reaction, Replication Accuracy, Stereolithography

\section{Introduction}

The observation of fallen snow crystals has been performed by microscopy ${ }^{(1-3)}$, and it was clarified that various types of snow crystals, such as needle, hexagonal plate, and dendrite types, are formed in the temperature range from $0{ }^{\circ} \mathrm{C}$ to $-20{ }^{\circ} \mathrm{C}$. The shapes of snow crystals are categorized in terms of the temperature and humidity of the atmosphere ${ }^{(4)}$. However, information obtained from microscopy is limited to plenary information, i.e., two-dimensional information, whereas three-dimensional (3D) information including profile heights has been acquired by drawing a 3D image visually obtained from microscopy. Rango et al. ${ }^{(5)}$, for example, observed snow crystals using a low-temperature scanning electron microscope and recorded the stereo images onto photographs. Stereo-paired microscopy has been popularized as an effective means of acquiring virtual 3D images in many fields, but the construction of 3D profile heights from a stereo-paired image is time-consuming work and the construction accuracy is poor for an intricate profile such as that of a snow crystal.

The method of snow crystal replication developed by Schaefer in $1941^{(6)}$ is a

*Received 31 Jan., 2012 (No. 12-0062) [DOI: 10.1299/jamdsm.6.923]

Copyright () 2012 by JSME 
convenient means of permanently preserving the shape of a snow crystal at room temperature, and the $3 \mathrm{D}$ profile of the snow crystal can be measured using a profilometer. In this method ${ }^{(6)}$, snow crystals are collected on the surface of a plastic solution and a volatile solvent included in the solution is evaporated in a cold and dry environment. However, the volatile solvents suitable for snow crystal replication are carcinogenic, poisonous, or ozone-depleting substances; therefore, this method is not in wide spread use. With this environmental background, the development of a sophisticated method for replicating snow crystals and measuring their 3D profiles is expected to promote the study of snow crystal growth mechanisms using crystallographic methods. In this paper, a new method is proposed for replicating snow crystals using a light-curing resin containing no harmful substances, as the replicating material, and the $3 \mathrm{D}$ reproduction of a snow crystal by stereolithography is reported.

\section{Experimental Methods}

\subsection{Replication of a snow crystal}

In the case of snow crystal replication using a plastic solution, the liquid replica must be kept in a cold and dry chamber to promote the volatilization of the solvent and to avoid the melting of the snow crystal, resulting in it taking over $6 \mathrm{~h}$ for the solidification. Another problem is that a secondary condensed snow crystal may become attached on the replica during the solidification process ${ }^{(7)}$. In this study, a light-curing resin is considered as an alternative to a plastic solution, and the possibility of shortening the solidification time, i.e., hardening time, is examined.

Figure 1 illustrates the proposed method for replicating snow crystals. First, a light-curing resin is smeared uniformly on a glass plate (a), and the glass plate is exposed to the atmosphere to collect a snow crystal (b). Next, a fluorescent light or a UV light is irradiated on the light-curing resin to solidify and harden the resin before the collected snow crystal begins to melt (c). These processes are carried out outdoors at a low atmospheric temperature. After the hardening of the light-curing resin, the replica is brought into a warm room and the snow crystal melts, evaporates and disappears in air (d). During the entire hardening process (c), the snow crystal floats on the liquid light-curing resin; therefore, one side of the snow crystal is replicated in the light-curing resin.

Because the temperature of the light-curing resin rises during the hardening process (c), the initial temperature of the glass plate and the light-curing resin in step (a) must be lower than $0{ }^{\circ} \mathrm{C}$. For example, if the expected temperature rise is $5{ }^{\circ} \mathrm{C}$, the initial temperature should be lower than $-5^{\circ} \mathrm{C}$. The temperature rise depends on the light-curing resin and the irradiation conditions.

Light-curing resins are categorized into two types ${ }^{(8)}$, those hardened by visual light irradiation and those hardened by UV light irradiation. The wavelengths of visual light are longer than those of UV light, resulting in a greater penetration depth of the light and greater thickness of the light-cured layer than those obtained by UV light irradiation. However, the irradiation energy of visual light is about half that of UV light; therefore, a longer hardening time is required for the former. The low energy of visual light has another disadvantage that the radicals that cause light hardening are trapped by oxygen in the atmosphere, resulting in reduced ability to harden the surface of the light-curing resin ${ }^{(8)}$.

In the case of UV light irradiation, the penetration depth is limited to several millimeters owing to its irradiation power being lower than that of visual light, but no deterioration of light-hardening ability occurs as a result of the trapping of radicals.

The key point in snow crystal replication is rapid processing, namely, the light-hardening reaction should be completed as quickly as possible. Also, the exposure of light-curing resin to the atmosphere is unavoidable when the replication method shown in Fig. 1 is adopted. A thickness of $1 \mathrm{~mm}$ for the light-cured layer is more than sufficient for 
replication because the thickness of a snow crystal is about $0.1 \mathrm{~mm}$. Taking these points into consideration, a light-curing resin that is hardened by UV light irradiation was selected as the material for snow crystal replication. The specifications of the light-curing resin are listed in Table 1. The light-curing resin, NOA81, is used as a transparent adhesive for glass and metals, and its main ingredients are polyene and polythiol.

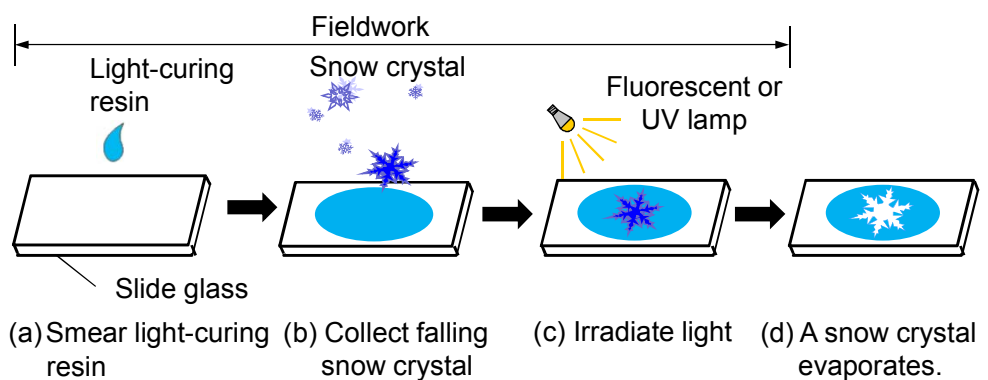

Fig.1 Replication of a snow crystal using a light-curing resin

Table 1 Light-curing conditions

\begin{tabular}{|l|l|}
\hline \multirow{3}{*}{ Light-curing resin } & $\begin{array}{l}\text { UV adhesive } \\
\text { Norland Products Inc., NOA81 } \\
\end{array}$ \\
& $\begin{array}{l}\text { Ingredients: polyene/polythiol } \\
\text { Viscosity: } 0.3 \mathrm{~Pa} \cdot \mathrm{s}\left(\text { at } 25^{\circ} \mathrm{C}\right) \\
\text { Quantity: } 20 \mu \mathrm{l}\end{array}$ \\
\hline UV Irradiation & $0.05-1.0 \mathrm{~mW} / \mathrm{cm}^{2}$ \\
\hline Irradiation time & $50 \mathrm{~min}$ \\
\hline Thermocouple & K-type, $\phi 50 \mu \mathrm{m}$ \\
\hline Room temperature & $20,10,0,-10,-20^{\circ} \mathrm{C}$ \\
\hline
\end{tabular}

\subsection{Characteristics of light-curing resin}

The light-hardening reaction of the light-curing resin generates heat, whose calorific value is markedly affected by the ingredients of the light-curing resin and the irradiation density of the light. When a snow crystal is replicated by the light-hardening reaction, it is essential to select irradiation conditions such that the temperature of the light-curing resin does not exceed $0{ }^{\circ} \mathrm{C}$ during the light-hardening process, which is the melting temperature of snow. A thermocouple was used to evaluate the temperature rise of NOA81.

Figure 2 illustrates the experimental setup. Twenty microliters of NOA81 resin, which is the same quantity as that used in the snow collection process, is smeared on the glass plate used for the collection of the snow crystal, and a K-type thermocouple is placed on the surface on which the snow crystal falls. The UV light irradiation density is given in Table 1.

It was reported ${ }^{(9)}$ that the temperature rise due to a light-hardening reaction depends on the initial temperature of the light-curing resin; for example, at a temperature of $-50{ }^{\circ} \mathrm{C}$, the ingredients of the light-curing resin only partly crystallize, resulting in a smaller temperature rise than that at room temperature. In the replication of a snow crystal, a lower temperature rise results in a more favorable collection condition; therefore, the experiments were performed in a chamber whose temperature can be controlled within $\pm 0.5^{\circ} \mathrm{C}$ to investigate the effect of temperature on the light-hardening characteristics. The viscosity of the light-curing resin, which changes with temperature and for which a high viscosity, i.e., a low fluidity, results in the deterioration of replication accuracy, was measured at various room temperatures by the line spread method ${ }^{(10)}$.

To evaluate the replication accuracy of the light-hardening process, a metal-surface roughness scale for contact probe profilometer (E-MC-S10A, TOKYO SEIMITSU CO., LTD.) was selected as the replication target in light-hardening experiments. 


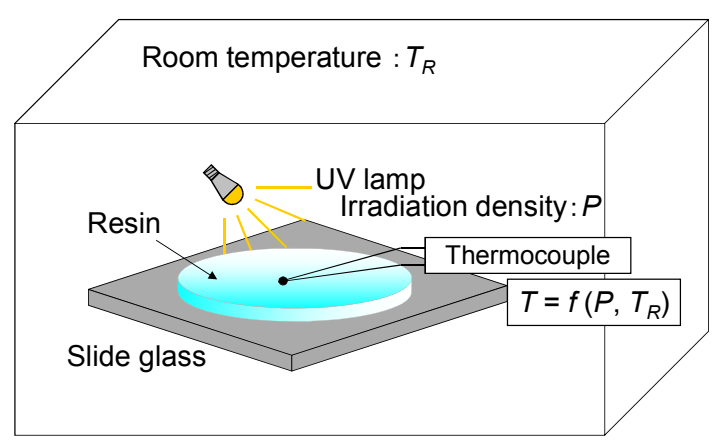

Fig.2 Setup for examining light-curing process

\subsection{D reproduction of a snow crystal}

The specifications of the stereolithography machine used in the 3D reproduction of the snow crystal are listed in Table 2. The diameter of the beam spot is $0.7 \mathrm{~mm}$ and the controllable minimum thickness of the light-curing resin is $0.1 \mathrm{~mm}$. The light-curing resin used in the stereolithography is an epoxy type (SOMOS8110) ${ }^{(11)}$, the mechanical properties of which are similar to those of polyethylene.

The 3D profile heights of the obtained snow crystal replica were measured using a confocal laser scanning microscope (LSM; Keyence Corporation, VK-9700/9710). The measurement accuracy of the LSM was $14 \mathrm{~nm}$ in the vertical direction and $20 \mathrm{~nm}$ in the horizontal direction. Because the snow crystal replica made of NOA81 is a transparent material, it is possible that the laser light may undergo interference with ambient laser light that penetrates into the replica and be reflected from the inside of the replica rather than from the surface. To eliminate the ambient laser light and improve the measurement accuracy, the surface of the replica was coated with Pt by sputtering before measurement.

Table 2 Experimental conditions of stereolithography

\begin{tabular}{|l|l|}
\hline \multicolumn{2}{|l|}{ Stereolithography machine UNIPAPID II (SP-1502) } \\
\hline Power source & UV lamp $\left(1.4 \mathrm{~mW} / \mathrm{cm}^{2}\right)$ \\
Beam spot diameter & $0.7 \mathrm{~mm}$ \\
UV light curing resin & Epoxy type (SOMOS8110) \\
Working range & $150 \times 150 \times 150 \mathrm{~mm}$ \\
Thickness of one layer & $0.1 \mathrm{~mm}$ \\
Scanning method & XY plot \\
\hline
\end{tabular}

\section{Experimental Results}

\subsection{Hardening process of light-curing resin}

Figure 3 shows the temperature rise $T$ plotted against the UV light irradiation time $t$ for various irradiation densities. The temperature rise due to light hardening occurs soon after the onset of UV light irradiation, and the temperature rise increases with increasing irradiation time regardless of the irradiation density. The temperature stops rising when the light-hardening reaction terminates then begins to fall to a constant temperature. The thermocouple placed on the surface of the light-curing resin measures the room temperature, which fluctuates depending on the on/off state of the thermostat in the room, the surface temperature of the light-curing-resin, which is warmed by the heat generated from the UV light itself, and the temperature rise due to the light-hardening reaction; therefore, the final temperature rise does not fall to zero but to a value depending on the UV light irradiation conditions. To define an accurate finishing time for the light-hardening reaction, the temperature gradient, i.e., the temperature change per $20 \mathrm{~s}$, was examined. 
Figure 4 shows the temperature rise-irradiation time curve and plots of the temperature gradient $T_{c}$ in the case of an irradiation density of $0.4 \mathrm{~mW} / \mathrm{cm}^{2}$, in which two parameters $T_{m}$ and $t_{f}$ are introduced to characterize the hardening process of the light-curing resin. The light-curing finishing time $t_{f}$ is defined as the time from the start of irradiation to the time at which the temperature change per $20 \mathrm{~s}$ takes a value of less than $0.1{ }^{\circ} \mathrm{C}$. The maximum temperature rise is denoted by $T_{m}$.

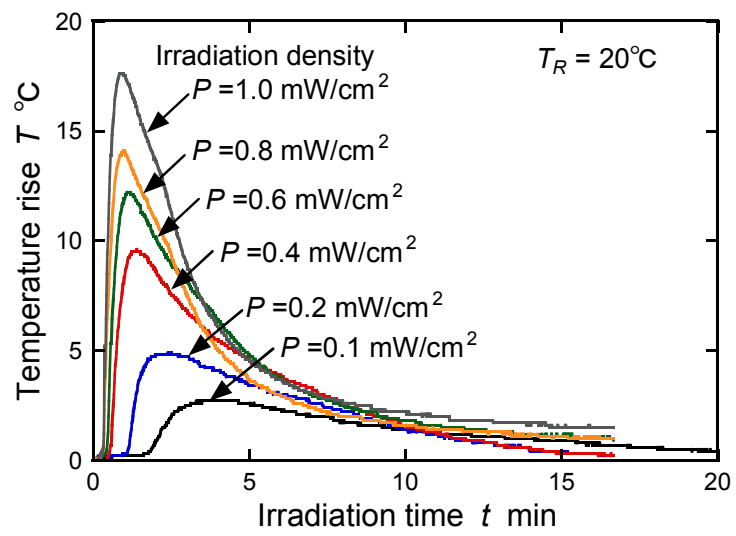

Fig.3 Temperature rise during light-curing process

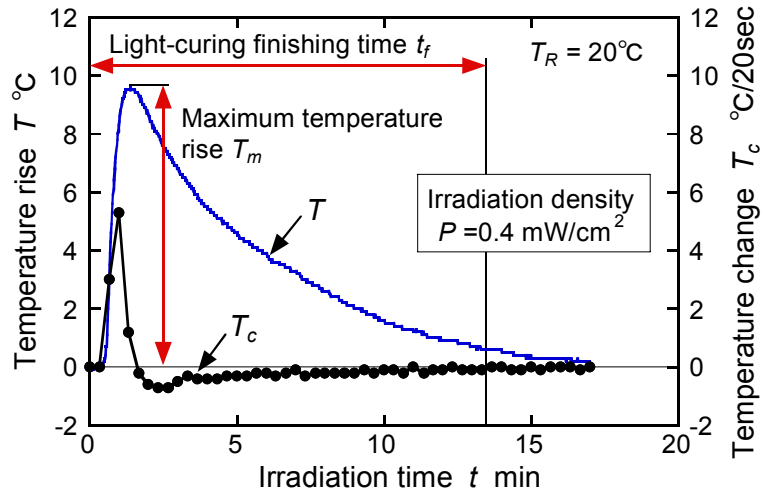

Fig.4 Parameters used to evaluate light-curing process

Figure 5 shows plots of the maximum temperature rise $T_{m}$ against the room temperature $T_{R}$ for various irradiation densities. The rate of the light-hardening reaction depends on the room temperature, and a low room temperature reduces the rate of light-hardening, resulting in a decrease in the maximum temperature rise. The results shown in Fig.5 clearly demonstrate this phenomenon. Examining the effect of the irradiation density, it can be seen that a higher irradiation density results in a higher maximum temperature for a given room temperature $T_{R}$.

Focusing on the maximum temperature rise at a room temperature of $0{ }^{\circ} \mathrm{C}$, by taking into consideration the fact that snow crystals are formed at an atmospheric temperature of lower than $0{ }^{\circ} \mathrm{C}$, it can be seen that the maximum temperature rise at an irradiation density of $1.0 \mathrm{~mW} / \mathrm{cm}^{2}$ is $4.2^{\circ} \mathrm{C}$.

This suggests that the temperature of the glass plate and the light-curing resin smeared on the glass plate before the collection of a snow crystal must be approximately $-5{ }^{\circ} \mathrm{C}$ to prevent the snow crystal from melting when an irradiation density of $1.0 \mathrm{~mW} / \mathrm{cm}^{2}$ is used for snow crystal replication. The results also suggest that a temperature of $-1.5{ }^{\circ} \mathrm{C}$ or lower is required when an irradiation density of $0.6 \mathrm{~mW} / \mathrm{cm}^{2}$ is applied.

Figure 6 shows plots of the light-curing finishing time $t_{f}$ against the irradiation density 
$P$. No light-hardening reaction occurs before the onset of UV irradiation; therefore, $t_{f}$ decreases in inverse proportion to the irradiation density at lower irradiation densities. No significant temperature rise occurred in some experiments carried out at irradiation densities of lower than $0.1 \mathrm{~mW} / \mathrm{cm}^{2}$; these data are not plotted in the figure. $t_{f}$ approaches a constant value of approximately $15 \mathrm{~min}$ at irradiation densities of $0.6 \mathrm{~mW} / \mathrm{cm}^{2}$ and above. It is desirable to select a short and constant irradiation time to complete the light-hardening process effectively. On this basis, an irradiation density of $0.6 \mathrm{~mW} / \mathrm{cm}^{2}$ or above is considered to be appropriate for UV light irradiation.

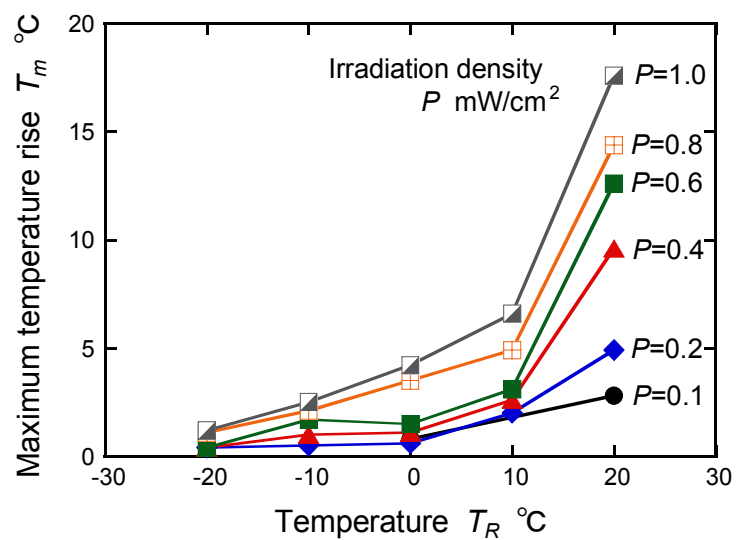

Fig.5 Effect of irradiation density on maximum temperature rise

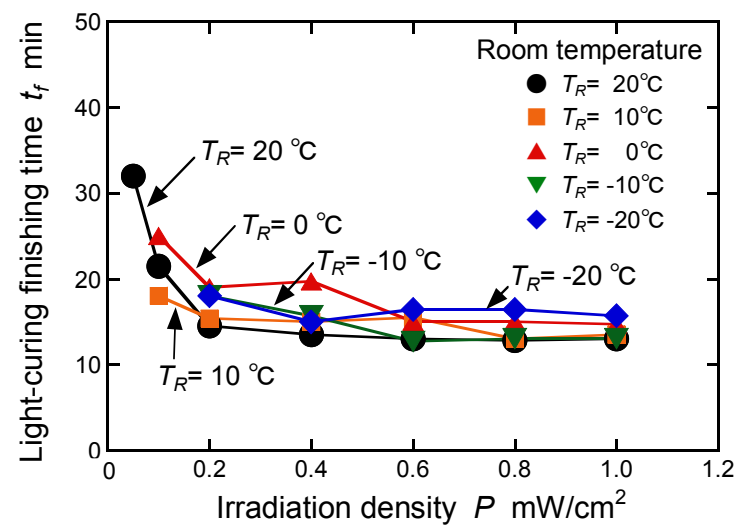

Fig.6 Effect of room temperature on light-curing finishing time

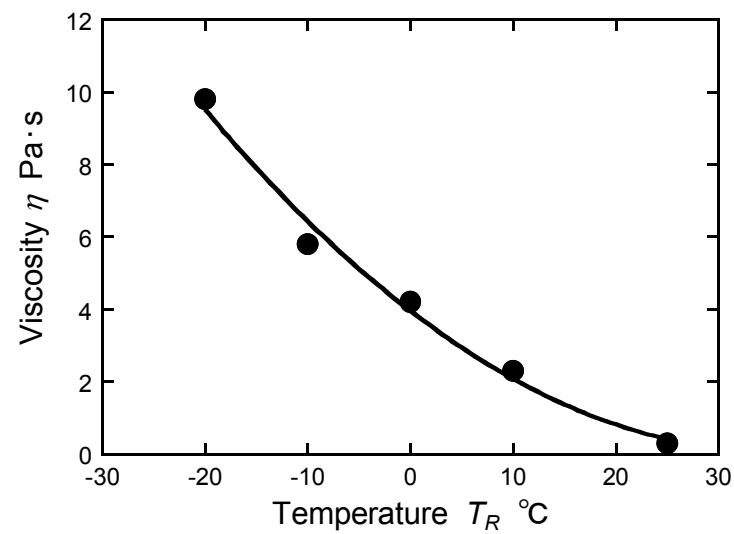

Fig.7 Effect of temperature on viscosity 


\subsection{Replication Accuracy}

Figure 7 shows plots of the viscosity $\eta$ of the light-curing resin NOA81 against the room temperature $T_{R}$. The viscosity markedly increases with decreasing room temperature, and the viscosity at $0{ }^{\circ} \mathrm{C}$ is more than 170 times that at a room temperature of $25^{\circ} \mathrm{C}$, which is adopted when NOA81 is used as an adhesive resin.

Figure 8 shows a LSM image and the sectional profile curve of the metal-surface roughness scale for contact probe profilometer (E-MC-S10A) (Fig.8 (a)), and those of replicas formed at room temperatures of $20,-10$, and $-20^{\circ} \mathrm{C}$ (Figs. 8 (b)-(d)).
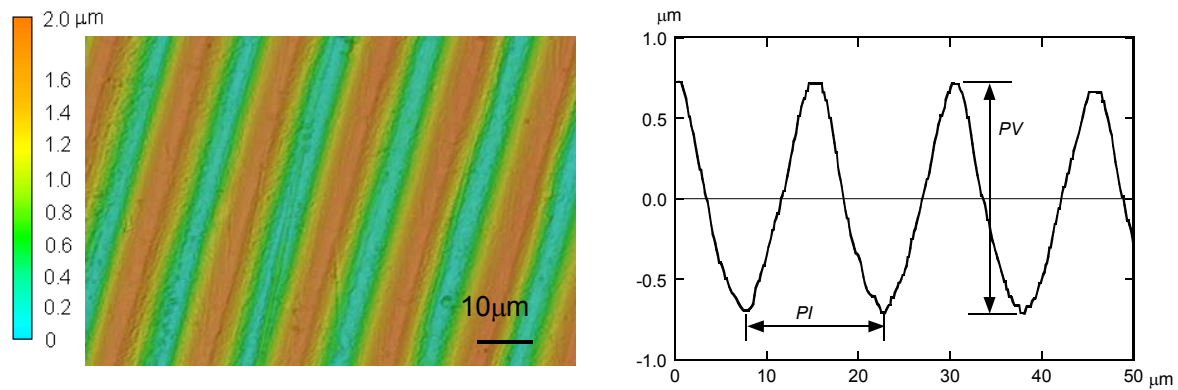

(a) Metal-surface roughness scale
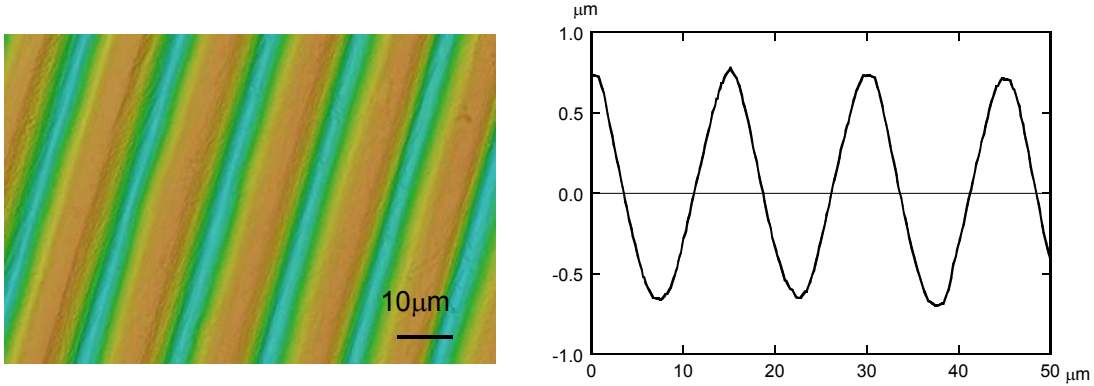

(b) Replica of metal-surface roughness scale at $20{ }^{\circ} \mathrm{C}$
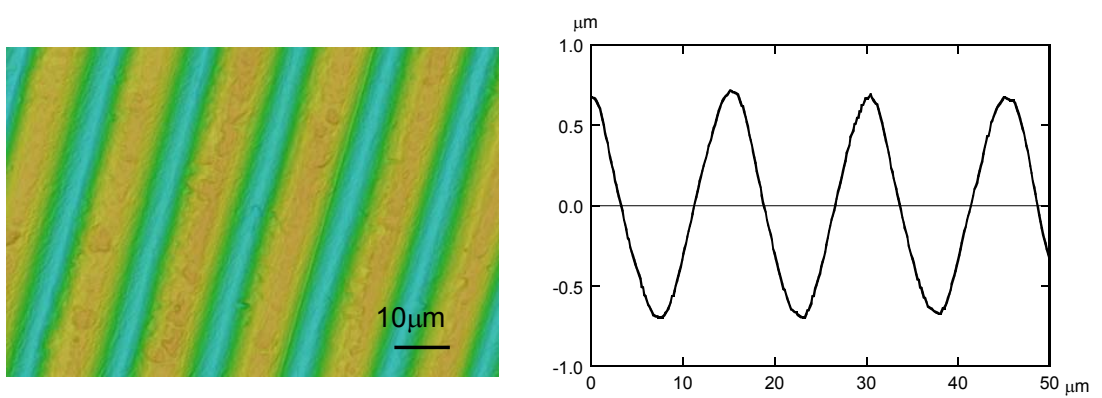

(c) Replica of metal-surface roughness scale at $-10{ }^{\circ} \mathrm{C}$
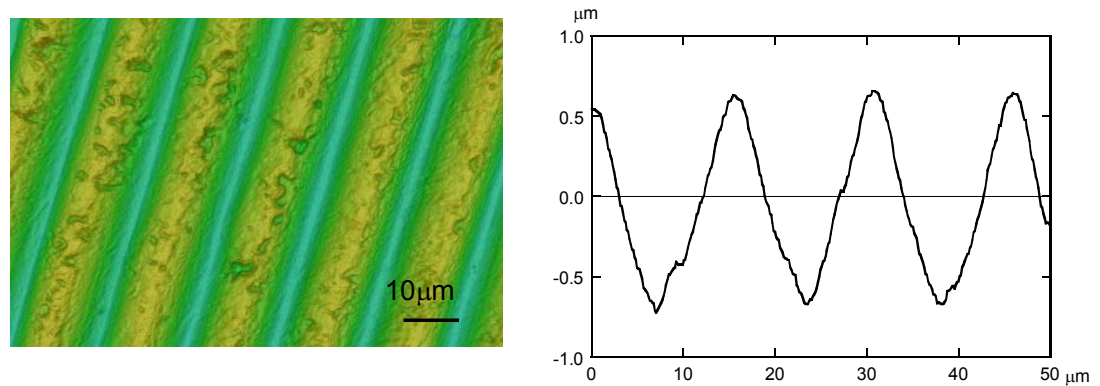

(d) Replica of metal-surface roughness scale at $-20{ }^{\circ} \mathrm{C}$

Fig.8 LSM images and profile curves of the surface roughness scale and the replicas 
The profile curve of the surface roughness scale is copied reversely onto the replica; therefore, the peaks and valleys of the profile curve shown in Fig.8 (a) correspond to the valleys and peaks of the profile curves shown in Figs.8 (b)-(d), respectively.

Examining the LSM images, some craters are observed on the top surface of the replica when the room temperature is $-10{ }^{\circ} \mathrm{C}$, as shown in Fig. 8 (c). The craters increase in number and spread over entire whole top surface when the room temperature is decreased to $-20{ }^{\circ} \mathrm{C}$, as shown in Fig.8 (d). The depths of craters are measured to be $0.15-0.65 \mu \mathrm{m}$. The existence of craters on the top surface of the replica means that the bottom surface of the surface roughness scale was not completely filled with the light-curing resin during the replication process owing to its high viscosity and low fluidity at subzero temperatures.

To evaluate the replication accuracy, three parameters were considered: the average pitch interval $P I$ and the average peak-to-valley value $P V$, shown in Fig.8 (a), and the resin filling rate $F$ defined by Eq.(1).

$$
F=\frac{\mathrm{A}_{t}-A_{c}}{A_{t}}=1-\frac{A_{c}}{A_{t}}
$$

Here, $A_{t}$ is the total area of the LSM image examined and $A_{c}$ is the total area of the craters observed on the LSM image.

Figure 9 shows plots of $P I, P V$, and $F$ against the room temperature $T_{R}$. The straight lines drawn in Figs.9 (a) and (b) indicate the average values of $P I$ and $P V$ for the surface roughness scale, respectively.

As shown in Fig.9 (a), the maximum difference in PI between the replicated profile and the original profile, i.e., the profile of the surface roughness scale, is $0.16 \mu \mathrm{m}(1.05 \%$ of the original $P I$ ) and no significant dependence of $P I$ on $T_{R}$ is found.

As shown in Fig.9 (b), the difference in $P V$ between the replicated profile and the original profile is $0.04 \mu \mathrm{m}(2.72 \%$ of the original $P V)$ at a room temperature of $20{ }^{\circ} \mathrm{C}$, for which the best replication accuracy was expected owing to the lowest viscosity of the light-curing resin NOA81. A possible explanation for this result is the decrease in volume of the light-curing resin after solidifying. The decrease in volume of NOA81 upon solidification is specified by the manufacturer to be $6 \%$, giving a linear decrease of $1.82 \%$ assuming that isotropic shrinkage occurs. The reason why this calculated value is smaller than the experimental value of $2.72 \%$ is that the shrinkage along the horizontal direction was inhibited by the friction between the surface roughness scale and the light-curing resin, resulting in increased shrinkage along the vertical direction. Focusing on the effect of the room temperature, $P V$ decreases with decreasing room temperature and is $0.08 \mu \mathrm{m}$ less than that of the surface roughness scale.

As shown in Fig.9 (c), $F$ decreases markedly when the room temperature is reduced to below $-10{ }^{\circ} \mathrm{C}$. This is due to the higher viscosity, i.e., the lower fluidity, of the light-curing resin at lower temperatures, and the shortage of the light-curing resin at the bottom of the surface roughness scale results in the formation of craters on the top surface of the replica, and the decrease in $P V$, as shown in Fig.9 (b).

From Fig. 9 it is clarified that $P V$ and $F$ are affected by the room temperature and that the replication accuracy deteriorates with decreasing room temperature. However, the depth of craters at the lowest room temperature of $-20^{\circ} \mathrm{C}$, at which the resin filling rate is lowest, is of sub-micron order and the change in the peak-to-valley value is less than $0.1 \mu \mathrm{m}$. It is also clarified that the shrinkage in the vertical direction, which affects the entire shape of the replica, is $2.7 \%$ for NOA 81 . 


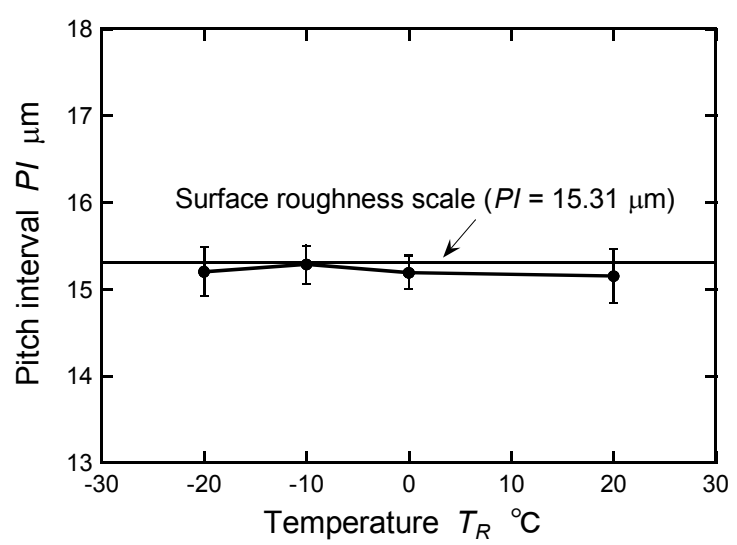

(a) Pitch interval

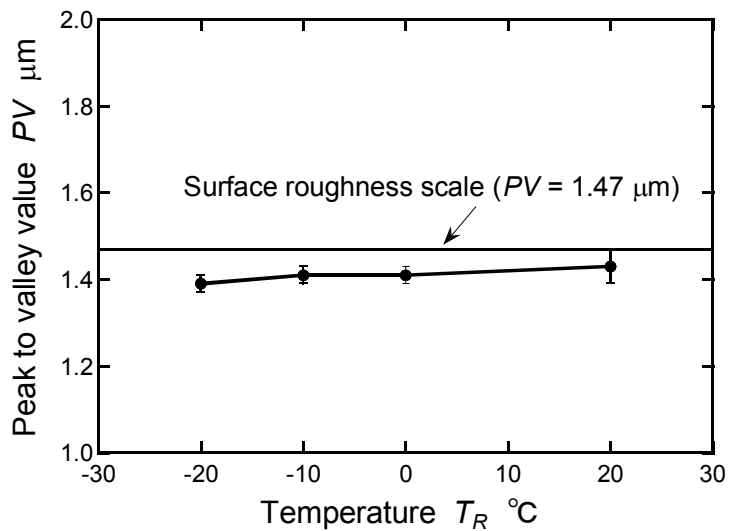

(b) Peak-to-valley value

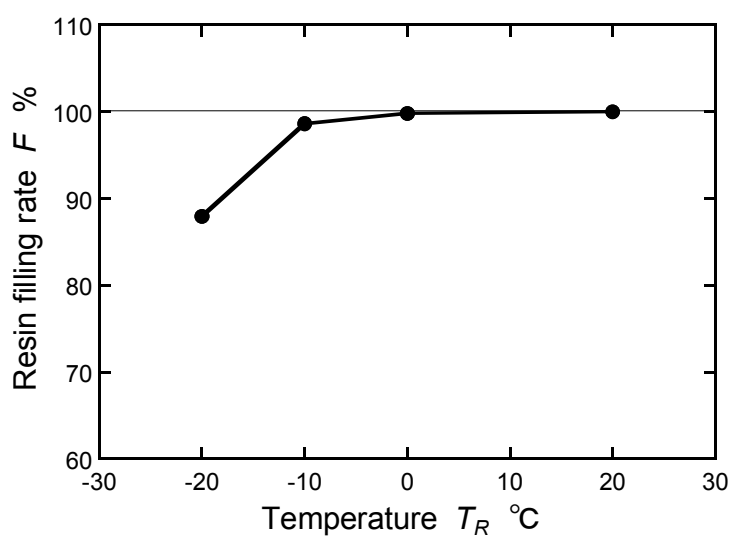

(c) Resin filling rate

Fig.9 Effect of temperature on replication accuracy

\subsection{D construction of a snow crystal}

Figure 10 shows an optical image of a snow crystal replica after Pt coating. The snow crystal was collected at Kamikawa town in Hokkaido at an atmospheric temperature of $-12{ }^{\circ} \mathrm{C}$. It can be seen that the snow crystal is a dendrite based on a regular hexagon with a diagonal of $1.48 \mathrm{~mm}$. The LSM has the ability to record the image of a frame as digital data with 2048 values in the $x$-direction and 1536 values in the $y$-direction with profile height data of 24 bits, i.e., $1 \mathrm{~nm}$ per digit. The size of a pixel is calculated to be $0.689 \mu \mathrm{m}$ in this case. The 3D profile data obtained from the LSM were input into a PC as CSV-formatted Excel data, and spike noises at the profile edges and ambient high-frequency noises were eliminated from the original data after reversing the original profile data. The size of data 
acceptable for an Excel file is limited to 512 lines; thus, the data acquired from the LSM were compressed to one-quarter of those of the LSM image, i.e., 512 values in the $x$-direction and 384 values in the $y$-direction.

The 3D profile data after noise elimination were transformed to DXF-formatted CAD data using free software; this format allows a maximum of 128 lines. This means that the 3D profile data after noise elimination should be compressed to one-quarter of those of the Excel file in the $x$-direction in order to process the data. The DXF-formatted CAD data were transformed to STL-formatted CAD data using Google SketchUp Pro8. The final number of CAD data was 121 in the $x$-direction and 115 in the $y$-direction, and the sampling interval was $13.78 \mu \mathrm{m}$.

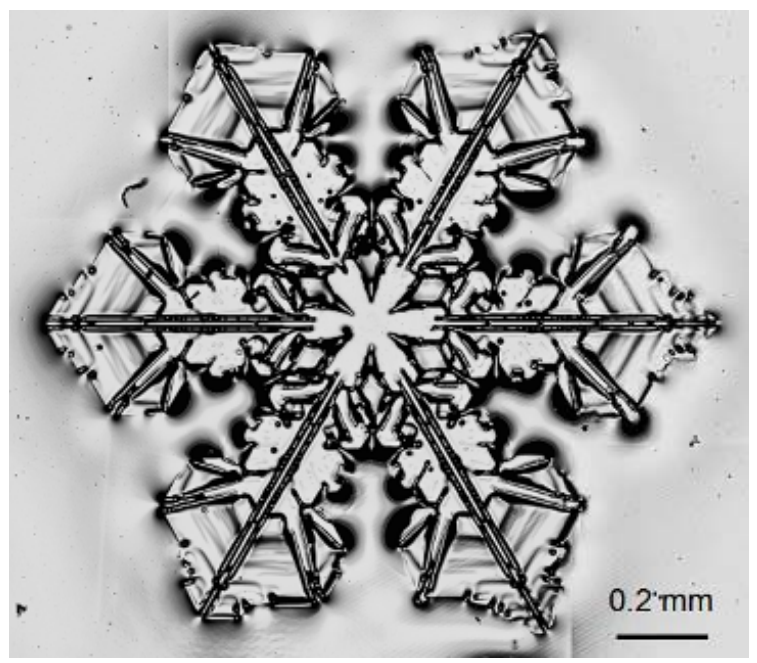

Fig.10 Optical image of a snow crystal replica

Figure 11 shows the 3D CAD model of the snow crystal shown in Fig.10 and its colored contour map. It was found from the contour map that the maximum height of the side of the snow crystal is $58 \mu \mathrm{m}$ and that it consists of a core, six main branches, and six twigs. The core has the form of a plateau of $45 \mu \mathrm{m}$ height with some projections above the plateau, each main branch has the form of a backbone with a height ranging from 25 to 40 $\mu \mathrm{m}$, and the twigs are about $10 \mu \mathrm{m}$ high. As shown in this contour map, the features of the snow crystal can be visually expressed with a contour line interval of a few microns; thus, it is concluded that the replication method using light-curing resin has sufficient replication accuracy to reconstruct the 3D shape of a snow crystal.

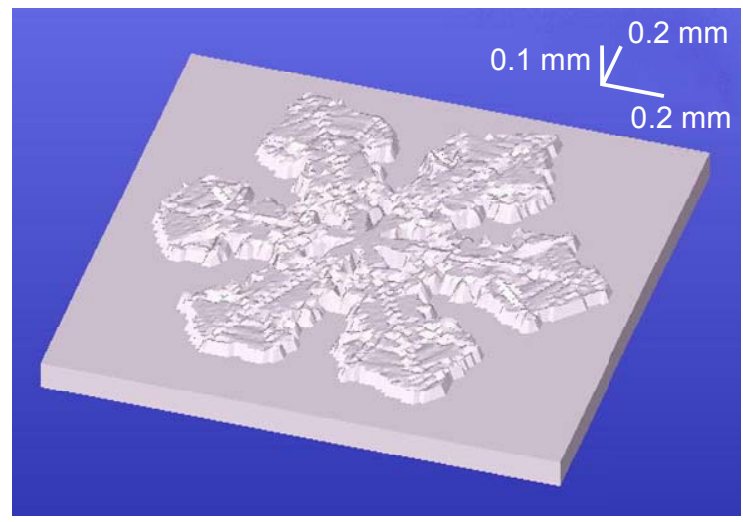

(a) 3D CAD model 


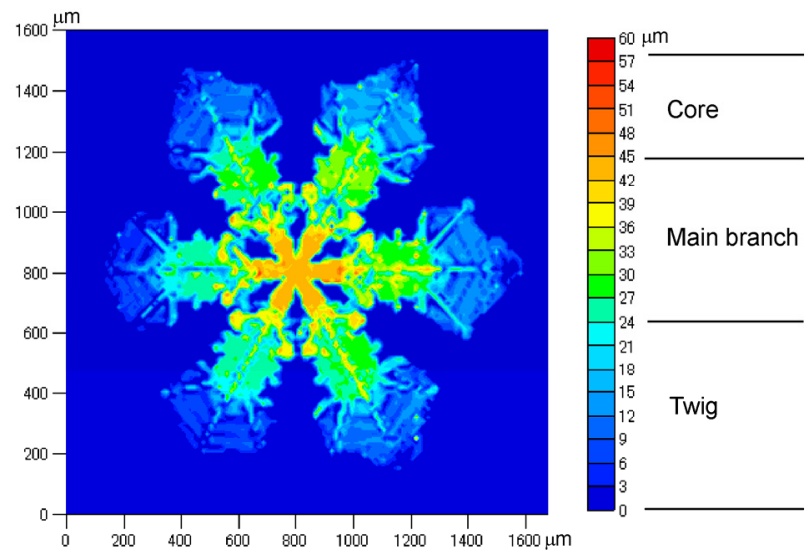

(b) Contour map

Fig.11 3D shape of a snow crystal

\subsection{Stereolithography of a snow crystal}

Figure 12 shows an optical image of the snow crystal reproduced by stereolithography. The magnification of the lithograph is 50 in both the vertical and horizontal directions. The lattice interval of the 3D profile data forming the lithograph is $0.689 \mathrm{~mm}$, which is almost the same as that of the beam spot diameter $(0.7 \mathrm{~mm})$. A comparison of the $3 \mathrm{D}$ reproduced snow crystal (physical model) shown in Fig.12 and the 3D CAD model (digital model) shown in Fig.11 reveals that the stereolithography faithfully copies the general feature of the original snow crystal.

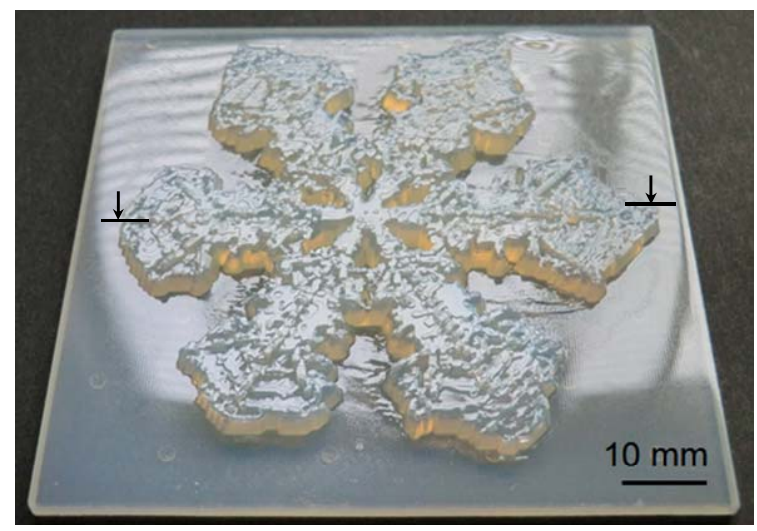

Fig.12 Optical image of a reproduced snow crystal

To evaluate the reproduction accuracy of the stereolithography process, the difference in the profile heights between the digital model and physical model was examined.

Figure 13 shows sectional profile curves of the main ridge indicated by the two arrows in Fig.12. Figure 13 (a) shows the profile curve of the physical model, i.e., the stereolithograph shown in Fig.12, and Fig.13 (b) shows the corresponding profile curve extracted from the digital model, i.e., the 3D CAD data. Examining the profile curves, it can be seen that the profiles are generally in agreement, but some of the sharp crests and shallow gorges, for example, those indicated by $\mathrm{A}, \mathrm{B}$, and $\mathrm{C}$ in the figure, are not reproduced in the physical model.

To measure the reproduction accuracy in a quantitative manner, the root-mean-square residual $R$ defined by Eq. (2) is used. 


$$
R=\sqrt{\frac{1}{N} \sum_{i=1}^{N}\left(Z_{C A D i}-Z_{R P i}\right)^{2}}
$$

Here, $Z_{C A D i}$ are the profile heights in the digital model, $Z_{R P i}$ are the profile heights in the physical model, and $N$ is the number of profile heights.

For our example, $R$ is calculated to be $0.27 \mathrm{~mm}$. Since the magnification of the stereolithograph is 50 , this value is converted to $5.4 \mu \mathrm{m}$ for the actual-size snow crystal replica shown in Fig.11. The reproduction accuracy of stereolithograhy depends on the diameter of the beam spot and the controllable minimum thickness. In the experiments, a beam spot diameter of $0.7 \mathrm{~mm}$ was used and the thickness of each layer was set to $0.1 \mathrm{~mm}$ to shorten the reproduction time. The reproduction accuracy can be improved by using a smaller beam spot and a thinner layer per scan.

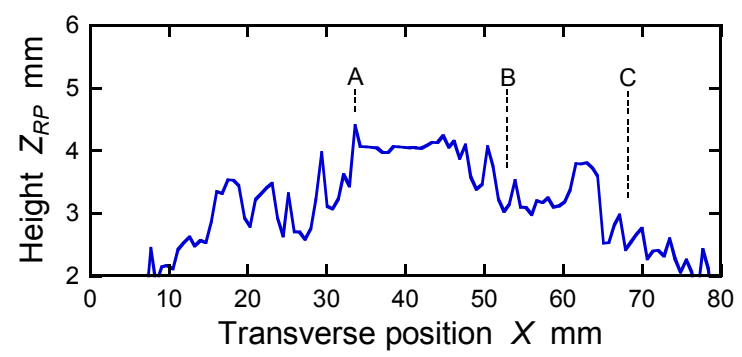

(a) Physical model (Stereolithograph)

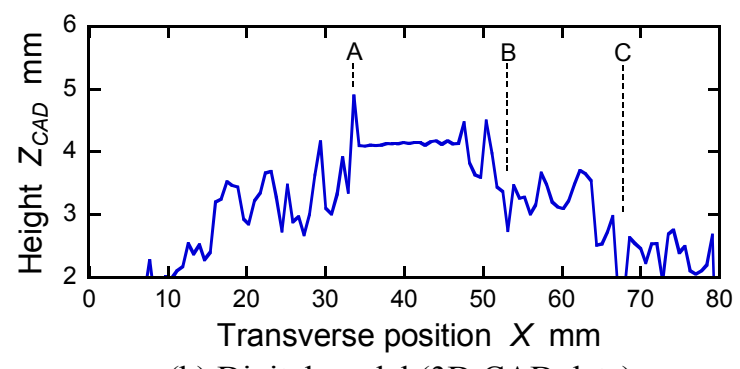

(b) Digital model (3D CAD data)

Fig.13 Profile of the main ridge of a snow crystal

\section{Conclusions}

To realize the 3D profile measurement of a snow crystal at room temperature, a method of replicating a snow crystal using a UV light-curing resin was developed. Moreover, the light-hardening process was examined for various values of atmospheric (room) temperature and UV light irradiation density. To evaluate the replication accuracy, a surface roughness scale was replicated using this method. Finally, to observe the features of the snow crystal without microscopy, the $3 \mathrm{D}$ reproduction of the snow crystal was conducted by stereolithography. The results are summarized as follows.

1) When the polyene/polythiol resin NOA81 was used as the replicating material, a UV light irradiation density of at least $0.6 \mathrm{~mW} / \mathrm{cm}^{2}$ was required to complete the light-hardening reaction within 15 min regardless of the atmospheric temperature.

2) The temperature of the light-curing resin increased owing to the light-hardening reaction, and the rate of the light-curing reaction decreases with decreasing atmospheric temperature. 3) When the atmospheric temperature was $0{ }^{\circ} \mathrm{C}$, the maximum temperature rise was $4.2{ }^{\circ} \mathrm{C}$ at an irradiation density of $1.0 \mathrm{~mW} / \mathrm{cm}^{2}$. This suggests that the temperature of the light-curing resin before the collection of a snow crystal must be approximately $-5^{\circ} \mathrm{C}$ when 
an irradiation density of $1.0 \mathrm{~mW} / \mathrm{cm}^{2}$ is applied at an atmospheric temperature of below $0{ }^{\circ} \mathrm{C}$.

4) The replication method had sufficient accuracy to reconstruct the 3D shape of a snow crystal.

5) The 3D reproduction of a snow crystal by stereolithography can be conducted by transforming the CSV-formatted 3D profile height data to STL-formatted CAD data, although the accuracy of reproduction depends on the stereolithography conditions.

\section{References}

(1) Nakaya, U., Snow Crystals: Natural and Artificial, (1954), pp.1-510, Harvard University Press, Cambridge, Massachusetts, USA.

(2) Magono, C. and Chung, W.L., Meteorological Classification of Natural Snow Crystals, Journal of the Faculty of Science, Hokkaido Univ., Ser.7, Vol.2, No.4 (1966), pp.321-335.

(3) Sommerfeld, R.A. and LaChapelle, E., The classification of snow metamorphism, Journal of Glaciology, Vol.9, No.55 (1970), pp.3-17.

(4) Bailey, M. P. and Hallett, J., A Comprehensive Habit Diagram for Atmospheric Ice Crystals, Journal of the Atmospheric Sciences, Vol.66 (2009), pp.2888-2899.

(5) Rango, A., Wergin, W.P., and Erbe, F.E., Snow crystal imaging using scanning electron microscopy: I. Precipitated snow, Journal of Hydrological Sciences, Vol.41, No.2 (1996), pp.219-233.

(6) Takahashi, T., and Fukuta N., Ice Crystal Replication with Common Plastic Solutions, Journal of Atmospheric and Oceanic Technology, Vol.5 (1988),pp.129-135.

(7) Yanagi, S, Application of light-curing resin to the preparation of falling snow and frost, Journal of Physics Education Society of Japan, Vol.53, No.1 (2005), pp.56-59 (in Japanese).

(8) Light-curing resin, Three Bond technical news, No.45 (1995), pp.1-8 (in Japanese).

(9) Ohta, R., Hosomi, H., Miyoshi, R. and Mitsuhashi, K., Analysis of hardening reaction of light-curing resin, The TRC News, No.108 (2009), pp.49-52 (in Japanese).

(10) Nicosia, M.A., Robbins, J., The Usefulness of the Line Spread Test as a Measure of Liquid Consistency, Dysphagia, Vol.22 (2007), pp.306-311.

(11) Hagiwara. T, Current status and trends of stereolithography resins, Journal of the Japan Society for Precision Engineering, Vol.70, No.2 (2004), pp.171-174 (in Japanese). 